\title{
Oxygen Reduction Electrocatalysis on Ordered Intermetallic Pd-Bi Electrodes is Enhanced by Low Coverage of Spectator Species
}

Yunfei Wang ${ }^{1+}$, Du Sun ${ }^{1+}$, Maoyu Wang ${ }^{2}$, Zhenxing Feng ${ }^{2}$ and Anthony Shoji Hall ${ }^{1 *}$

${ }^{1}$ Department of Materials Science and Engineering, Johns Hopkins University, Baltimore, Maryland 21218

${ }^{2}$ School of Chemical, Biological, and Environmental Engineering, Oregon State University, Corvallis, Oregon 97331

+ Denotes co-first authors

KEYWORDS: electrocatalysis, ordered intermetallic, alloy, oxygen reduction reaction, electrodeposition

\section{Table of Contents}

Figures and Tables

Figure S1. Temperature dependent oxygen reduction electrocatalysis. ............................................... 2

Figure S2. Near-edge X-ray Adsorption Near Edge Structure (XANES) of electrodes. .......................... 2

\section{Materials and Methods}

Materials.

Methods

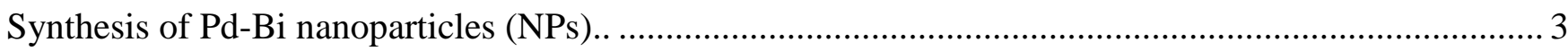

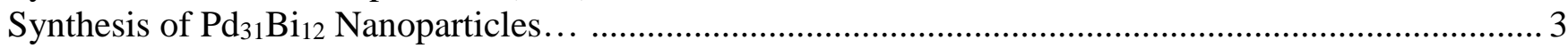

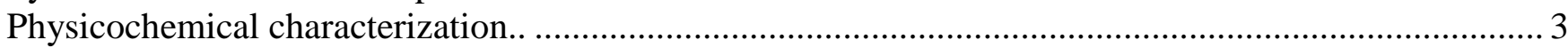

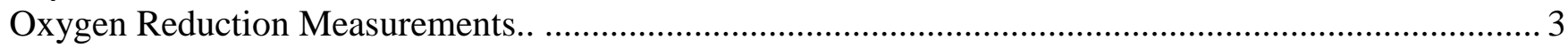

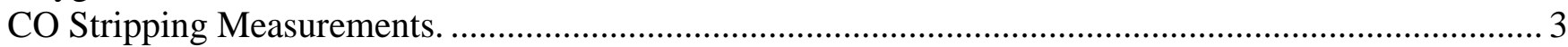

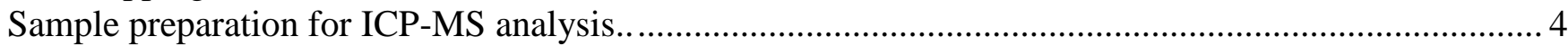

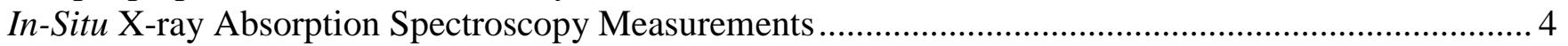

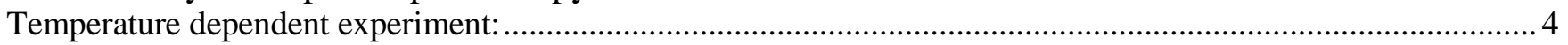

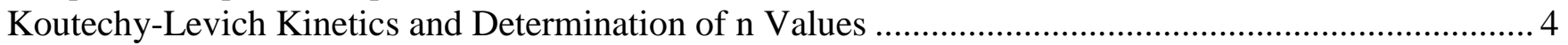

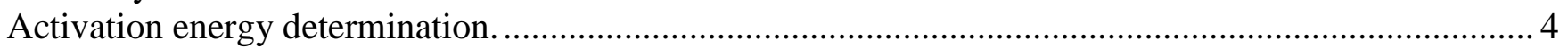




\section{Figures and Tables}

a)

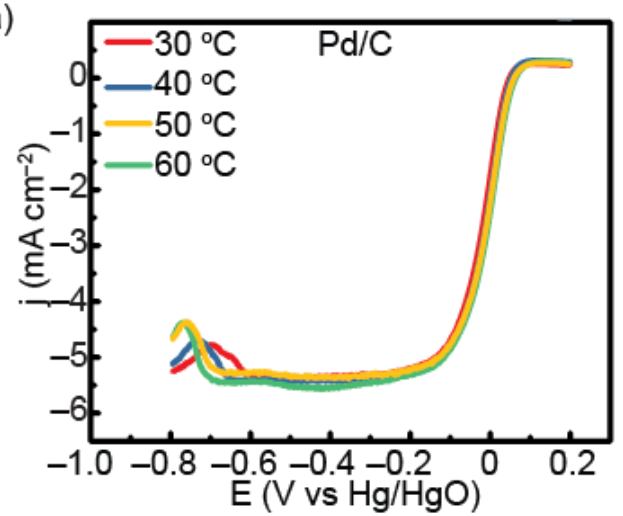

c)

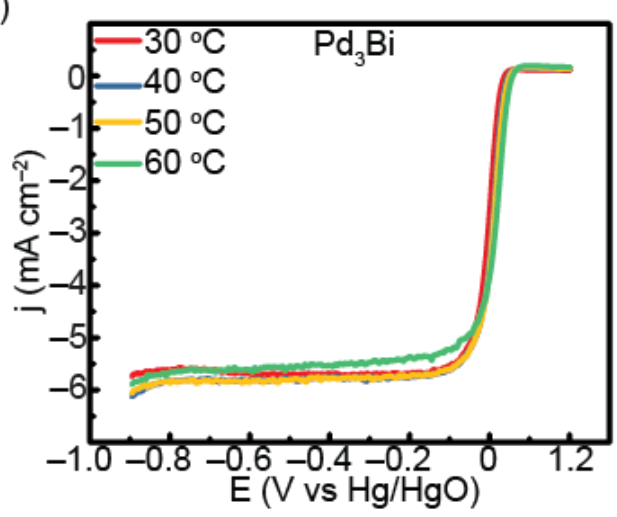

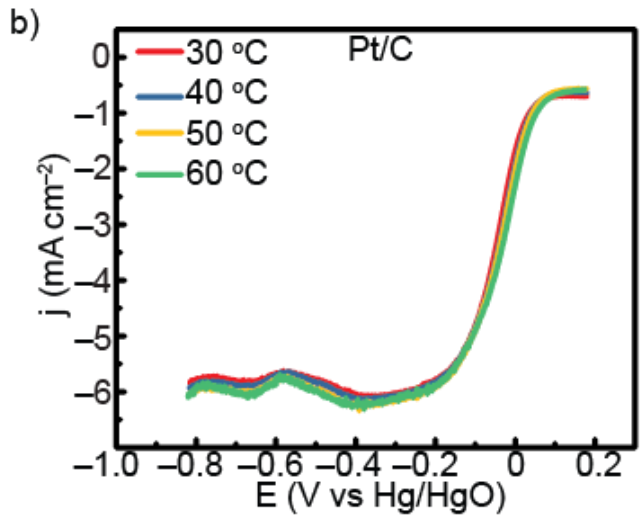

d)

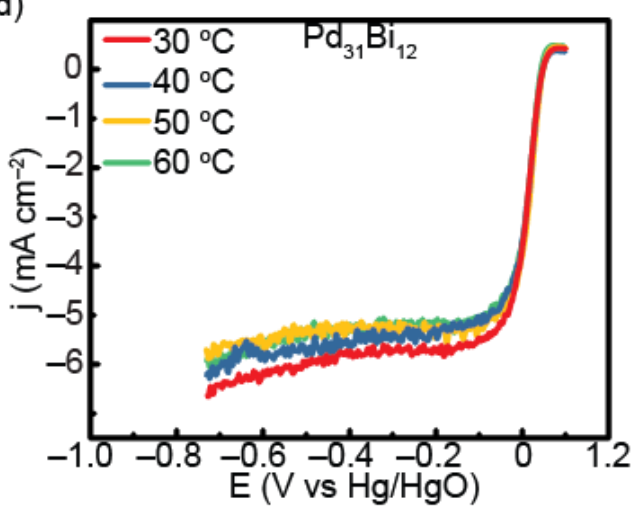

Figure S1. Temperature dependent oxygen reduction electrocatalysis measured on a) $\mathrm{Pd} / \mathrm{C}, \mathrm{b}) \mathrm{Pt} / \mathrm{C}, \mathrm{c}) \mathrm{Pd}{ }_{3} \mathrm{Bi}$, and d) $\mathrm{Pd}_{31} \mathrm{Bi}_{12}$.
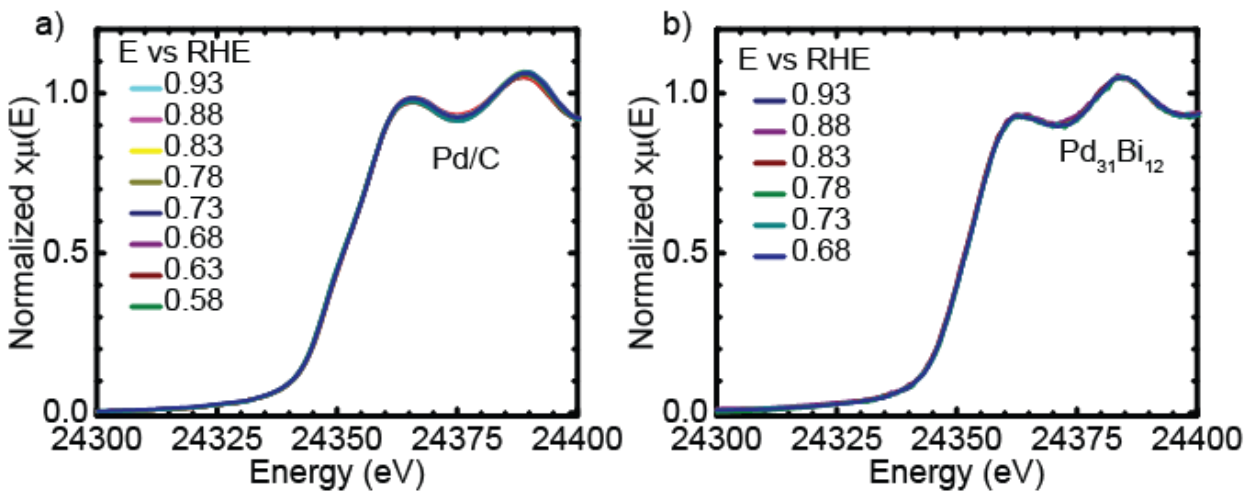

Figure S2. Near-edge X-ray Adsorption Near Edge Structure (XANES) of the Pd K-edge for Pd/C and $\mathrm{Pd}_{31} \mathrm{Bi}_{12}$ nanoparticles. 


\section{Experimental Methods}

Materials. Palladium (II) acetylacetonate (Pd(acac)2, 99\%, Strem Chemicals), Bismuth(III) acetate (Bi(oac)3, 99.99\%, Alfa Aesar), and oleylamine (OLAM, technical, 70\%, Aldrich), 40\% Pd on Vulcan XC-72 (Premetek Co), O2 gas (UHP grade, Airgas), 40\% Pt on High Surface Area Carbon (TEC10E40E, Tanaka Kikinzoku Kogyo TKK), were used as received without purification. All syntheses were carried out under $\operatorname{Ar}(\mathrm{g})$ using standard Schlenk techniques, and work-up proce-dures were performed in air. Electrolyte solutions were prepared with deionized water (resistance: $18.2 \mathrm{M} \Omega$ ) and potassium hydroxide (KOH, Semiconductor Grade 99.99\%, Alfa Aesar).

Synthesis of PdBi 2 Nanoparticles (NPs). In a typical synthesis, $61 \mathrm{mg}$ of $\mathrm{Pd}(\mathrm{acac})_{2}(0.2 \mathrm{mmol}), 154 \mathrm{mg}$ of Bi $(\mathrm{oac})_{3}(0.4 \mathrm{mmol})$, and $10 \mathrm{~mL}$ of OLAM were first added into a $20-\mathrm{mL}$ scintillation vial with a magnetic stir bar. After stirring for $20 \mathrm{mins}$, the vial was sonicated for another $10 \mathrm{mins}$ to ensure all the solid precursors were dissolved and the mixture formed a pale-yellow solution. Another $5 \mathrm{~mL}$ of OLAM were added into a 100-mL 3-neck round-bottom flask with a condenser, thermometer adapter, thermometer, and rubber septum. Magnetic stirring was started, and the solution was degassed under vacuum at $120^{\circ} \mathrm{C}$ for $\sim 5-10$ minutes. The flask was backfilled with Ar and heated to $\sim 300^{\circ} \mathrm{C}$ at a rate of $10^{\circ} \mathrm{C} / \mathrm{min}$. Once the temperature reached $300^{\circ} \mathrm{C}$, the $10 \mathrm{~mL}$ of precursor solution was slowly injected into the flask using a syringe pump at a rate of $0.3 \mathrm{~mL} / \mathrm{min}$. The solution in the flask turned from colorless to light brown and finally formed a dark brown colloidal mixture when the injection was completed. The solution was then cooled rapidly by removing the flask from the heating mantle. The nanoparticles were precipitated by adding $10 \mathrm{~mL}$ of ethanol and then centrifuged at $8000 \mathrm{rpm}$ for $5 \mathrm{~min}$. The precipitate was washed three times using a 1:1 ethanol/toluene mixture (with centrifugation in between washes) and could then be suspended in ethanol for further characterization and electrode preparation.

Synthesis of Pd $\mathbf{3}_{11} \mathbf{B i}_{12}$ Nanoparticles. The Vulcan carbon was suspended in IPA and water (1:1 mixture by vol.) by sonication, then drop casted on the glassy carbon electrode with a loading of $\sim 0.38 \mathrm{mg} / \mathrm{cm}^{2} . \mathrm{Pd}_{31} \mathrm{Bi}_{12}$ ordered intermetallics grown directly onto the carbon particles by deposition from an aqueous electrolyte containing $50 \mathrm{mM}$

Ethylenediammenetetracetic acid (EDTA), $25 \mathrm{mM} \mathrm{Bi}\left(\mathrm{C}_{2} \mathrm{H}_{3} \mathrm{O}_{2}\right)_{3}$ and $4 \mathrm{mM} \mathrm{Pd}\left(\mathrm{NO}_{3}\right)_{2}$ at $30{ }^{\circ} \mathrm{C}$. The pulse-on potential was chosen to be $-0.35 \mathrm{~V}$ vs Normal Hydrogen Electrode (NHE, henceforth all potentials will be referenced to this), which is higher than the minimum potential to deposit Pd or Pd-Bi alloy to supply the necessary overpotential for fast nucleation. The pulse-on time was optimized to $30 \mathrm{~ms}$ so that the nuclei can form, the pulse-reverse was chosen at $0.65 \mathrm{~V}$ for $5 \mathrm{~s}$ is allow the local Pd cation concentration to relax to equilibrium, providing a uniform concentration profile during the on pulse, thereby decreasing the spatial variations of the potential distribution. ${ }^{1}$ After 200 pulse cycles, chronoamperometry at $-0.1 \mathrm{~V}$ is applied for $180 \mathrm{~s}$ to grow the deposited nuclei into ordered intermetallic $\mathrm{Pd}_{31} \mathrm{Bi}_{12}$, during this step a current density around $0.5 \mathrm{~mA} / \mathrm{cm}^{2}$ was observed. The as-deposited sample was cleaned to remove organic residue as described in our previous article..$^{2-3}$

Physicochemical characterization. The ethanol suspension of the nanoparticles was dropcasted onto a zero-background $\mathrm{Si}$ wafer substrate and examined with a Philips X'Pert Pro Powder X-Ray diffractometer (XRD) with CuK radiation $\left(K \alpha_{1}, \lambda=1.5406 \AA\right.$ and $\mathrm{K \alpha}_{2}, \lambda=1.5444 \AA$ ). Transmission electron microscopy (TEM) was performed on a FEI F200C Talos or FEI Tecnai TF30 operation at $200 \mathrm{kV}$. The materials on the glassy carbon disk after electrochemical test was dissolved by concentrated nitric acid and then diluted with water. The metal concentration in the nitric acid solution is analyzed by the PerkinElmer NexION 300D with ICP.

Oxygen Reduction Measurements. Electrochemical measurements were carried out with a potentiostat/galvanostat (Metrohm Autolab Potentiostat or Nuvant EzStat Pro) and a three-electrode Teflon cell with $0.1 \mathrm{M} \mathrm{KOH}$ aqueous solution as the electrolyte. A $\mathrm{Hg} / \mathrm{HgO}$ electrode in $1 \mathrm{M} \mathrm{NaOH}$ was used as the reference electrode. A graphite rod was used as the counter electrode. $\mathrm{The} \mathrm{Hg} / \mathrm{HgO}$ reference electrode was periodically checked with a Reversible Hydrogen Electrode (RHE) to verify the stability of the reference potential. All data measured with IR compensation on the potentiostat. ORR was performed by sweeping the voltage from $-0.9 \mathrm{~V}$ to $0.2 \mathrm{Vvs.} \mathrm{Hg} / \mathrm{HgO}$ at a sweep rate of $20 \mathrm{mv} / \mathrm{s}$, or by constant potential chronoamperometery within the same potential range. All measurements were performed on a $5 \mathrm{~mm}$ diameter rotating disk electrode at $1600 \mathrm{rpm}$. All ORR measurments were IR-corrected, the IR drop was measured by the positive feedback or current interrupt method.

ECSA Measurement by CO Stripping. We utilized the CO stripping method to determine the electrochemically active surface area (ECSA) of Pt or Pd atoms on the surface of the electrode. The electrodes are first held at $0.15 \mathrm{~V}$ vs RHE for $20 \mathrm{~min}$ in $0.1 \mathrm{M} \mathrm{KOH}$ saturated with $\mathrm{CO}$ to form a surface adsorbed $\mathrm{CO}$ monolayer, followed by removal of $\mathrm{CO}$ (aq) by saturating the electrolyte with $\mathrm{Ar}$ for $15 \mathrm{~min}$. Finally, a the voltage was swept from $0.15 \mathrm{~V}$ to $1.15 \mathrm{~V}$ vs RHE to oxidize the adsorbed CO layer, the surface area of the sample can be calculated by integrating the oxidation peak assuming one $\mathrm{CO}$ adsorbed per $\mathrm{Pd}$ or $\mathrm{Pt}$ atom on the surface. The ratio of charge 
transferred for CO oxidation and metal surface area is estimated as $484 \mu \mathrm{C} / \mathrm{cm} 2$ for Pt and $420 \mu \mathrm{C} / \mathrm{cm} 2$ for Pd and $\mathrm{Pd}_{3} B i$. We expect that $\mathrm{Bi}$ sites will not adsorb $\mathrm{CO}$, therefore $\mathrm{CO}$ stripping will not account for catalytically inactive $\mathrm{Bi}$ sites on the surface.

Conversion of $\mathbf{P d B i}_{2}$ to $\mathbf{P d}_{3} \mathbf{B i}$. The electrode was prepared by directly dropcasting $4 \mu \mathrm{L}$ of the ethanol suspension of the as-prepared nanoparticle onto the glassy carbon disk. After it dried out, the electrode was then immersed in $0.1 \mathrm{M}$ acetic acid solution for 20 mins followed by DI water wash, acetone wash, and ethanol wash. Finally, the electrode was placed in a UV-ozone chamber and treated with $\mathrm{UV}$ and ozone at room temperature for $20 \mathrm{mins}$. A graphite rod and $\mathrm{Hg} / \mathrm{HgO}$ electrode were used as the counter and reference electrodes, respectively. The working electrode was then conditioned by cycling the potential from $-0.9 \mathrm{~V}$ to $0.2 \mathrm{~V} \mathrm{vs}$. $\mathrm{Hg} / \mathrm{HgO}$ reference at a scan rate of $0.1 \mathrm{~V} \mathrm{~s}^{-1}$ and at a rotation rate of $1600 \mathrm{rpm}$ for $\sim 500$ cycles.

Preparation of Pt/C and Pd/C electrodes. To prepare Pd/C materials, $30 \mathrm{mg}$ of $40 \% \mathrm{Pd}$ on Vulcan XC 72 (Premetek Co) was dispersed into a solution consisting of a mixture of $8 \mu \mathrm{l}$ Nafion Ionomer, $1 \mathrm{ml}$ DI water and $1 \mathrm{ml}$ IPA. The resulting solution was sonicated for $20 \mathrm{~min}$. $1 \mu \mathrm{l}$ of the mixture was drop casted onto the $5 \mathrm{~mm}$ diameter disk. The $\mathrm{Pt} / \mathrm{C}$ sample is prepared with the same procedure except with $\mathrm{Pt} / \mathrm{C}$ (TKK).

Sample preparation for ICP-MS analysis. After the electrochemical measurement, the glassy carbon electrode was taken out of the electrolyte and rinsed with $18.2 \mathrm{M} \Omega$ water. After dried in air, the electrode was placed in a plastic tube and $1 \mathrm{~mL}$ concentrated TraceMetal grade nitric acid was added into the tube. The tube was sonicated for a few minutes to fully dissolve the catalyst on the surface. Additional $9 \mathrm{~mL}$ of $18.2 \mathrm{M} \Omega$ water was added to the tube and the solution is ready for testing.

In-Situ X-ray Absorption Spectroscopy Measurements: In-situ X-ray absorption near edge structure (XANES) experiments were performed at beamline 2-2 at Stanford Synchrotron Radiation Lightsource (SSRL) of SLAC National Accelerator Laboratory. $2 \mathrm{mg}$ catalyst was deposited onto a $100 \mu \mathrm{m}$ thick and $2.5 \times 1.5 \mathrm{~cm}^{2}$ carbon fiber paper to form a $0.5 \times 1 \mathrm{~cm}^{2}$ active area as the working electrodes. The working electrodes, counter electrodes $(\mathrm{Pt})$ and reference electrodes $(\mathrm{Ag} / \mathrm{AgCl})$ were mounted onto a customdesigned in-situ XAS fluorescence cell filled with 0.1M KOH. (Cited: Wang et al., Nano-Micro Letters, 11, 47, 2019.) All the electrochemical measurement was done by a CHI 760E electrochemical workstation with oxygen gas flow. A Lytle detector was used to collect Pd K-edge fluorescence signal. All XAS data analysis were performed with Athena to extract XANES information. ${ }^{4}$

\section{Temperature dependent experiments:}

The electrochemical cell was placed in a water bath equipped with programmable heating unit. A Teflon-coated thermometer was also used to monitor the liquid temperature inside the cell. In a typical measurement, reference electrode and counter electrode were first assembled to the cell. The water bath temperature was then set to a certain value so that the cell temperature can reach the target temperature $\left(30,40,50\right.$, or $\left.60^{\circ} \mathrm{C}\right)$ after equilibrium. Noted that the set temperature is higher than the target temperature due to the heat loss of the system to the environment. The electrolyte was sparged with $\mathrm{O}_{2}$ while waiting for the temperature to increase. Once the temperature was stabilized at the target value, the working electrode was immersed into the electrolyte and a standard LSV was recorded at the same rotation rate $(1600 \mathrm{rpm})$. The potential of the reference electrode at difference temperature was determined by the equation given in the literature. ${ }^{5}$

\section{Koutechy-Levich Kinetics and Determination of $\mathbf{n}$ Values}

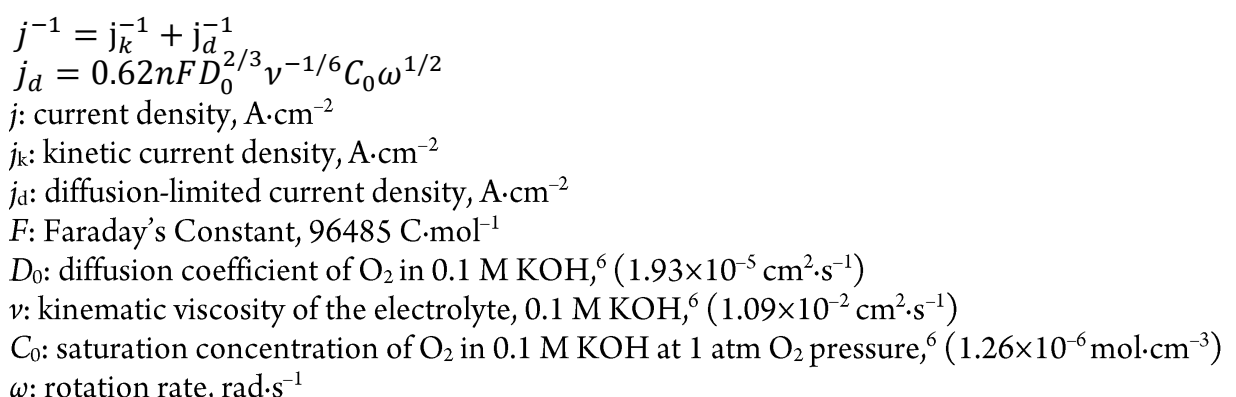

Taking $0.5 \mathrm{~V}$ as an example, the linear fitting of the plot of $j^{-1} \mathrm{vs} \omega^{-1 / 2}$ (figure $4 \mathrm{~b}$ ) has a slope of $2205 \mathrm{~A}^{-1} \cdot \mathrm{cm}^{2} \cdot \mathrm{s}^{-1 / 2} \cdot \mathrm{rad}^{1 / 2}$, which corresponds to $n=3.94$.

Activation energy determination. Quantitative data for the ORR kinetics were obtained from mass-transfer-corrected plots of the overpotential vs $\log j$, shown in Figure SI12, we observe two linear regions with different slopes. The slopes from each linear region 
corresponding to different temperatures are summarized in table S2. Arrhenius plot of $\boldsymbol{l o g} \boldsymbol{j}_{\boldsymbol{k}}$ vs. $\mathbf{1} / \boldsymbol{T}$ were constructed for a specific overpotential $(\boldsymbol{\eta})$, where $\boldsymbol{i}_{\boldsymbol{k}}$ is the mass transport corrected current density (kinetic current density) and $\boldsymbol{T}$ is the temperature. The $\boldsymbol{\eta}$ is calculated by equation 1, where the reversible oxygen reduction potential $\left(\boldsymbol{E}_{\boldsymbol{r}}\right)$ at each temperature is determined by the equations 2 and 3. The $\log \boldsymbol{j}_{\boldsymbol{k}}$ associated with that $\boldsymbol{\eta}$ is obtained by interpolation of the current at a fixed overpotential from the data shown in Figure SI12. The activation energy $\left(\boldsymbol{E}_{\boldsymbol{a}}\right)$ at this $\boldsymbol{\eta}$ can then be determined by the slope of the Arrhenius plot (equation 4$)$.?

$\eta=E_{r}-E(\mathrm{~V})$

$E_{r}=-\Delta G^{0} / n F(\mathrm{~V})(2)$

$\Delta \mathrm{G}^{0}=-295.6-0.03347 \mathrm{~T} \ln \mathrm{T}+0.3884 \mathrm{~T}(\mathrm{~kJ} / \mathrm{mol})(3)$

$E_{a}=-2.303 R\left[d \log j_{k} / d(1 / T)\right](\mathrm{kJ} / \mathrm{mol}) \quad(4)$

In the equations listed above, $\mathrm{E}$ is the applied potential, $\Delta G^{0}$ is the free energy for the $\mathrm{O}_{2}$ and $\mathrm{H}_{2}$ reaction producing liquid $\mathrm{H}_{2} \mathrm{O}, \mathrm{n}$ is the number of electrons transferred to producing on mole of water, which is 2 , and $\mathrm{F}$ is the Faraday constant.

\section{References}

1. Lee, J.-M.; West, A. C., Impact of pulse parameters on current distribution in high aspect ratio vias and through-holes. J. Electrochem. Soc. 2005, 152, C645.

2. Wang, Y.; Sun, D.; Chowdhury, T.; Wagner, J. S.; Kempa, T. J.; Hall, A. S., Rapid room-temperature synthesis of a metastable ordered intermetallic electrocatalyst. J. Am. Chem. Soc. 2019, 141, 2342-2347.

3. Wang, Y.; Hall, A. S., Pulsed electrodeposition of metastable $\mathrm{Pd}_{31} \mathrm{Bi}_{12}$ nanoparticles for oxygen reduction electrocatalysis. ACS Energy Lett. 2020, 5, 1, 17-

4. Ravel, B.; Newville, M., Athena, artemis, hephaestus: Data analysis for Xx-ray absorption spectroscopy using ifeffit. J. Synchrotron Radiat.Journal of Synchrotron Radiation 2005, 12, 537-541.

5. Case, B.; Bignold, G. J., The mercury/mercuric oxide electrode in high temperature aqueous solutions. J. Appl. Electrochem. 1971, 1, 141-146.

6. Min, X.; Chen, Y.; Kanan, M. W., Alkaline o2 reduction on oxide-derived au: High activity and 4e- selectivity without (100) facets. Phys. Chem. Chem. Phys. 2014, 16, 13601-13604

7. Parthasarathy, A.; Srinivasan, S.; Appleby, A. J.; Martin, C. R., Temperature dependence of the electrode kinetics of oxygen reduction at the platinum/nafion ${ }^{\circledR}$ interface—a microelectrode investigation. J. Electrochem. Soc. 1992, 139, 2530-2537. 